Секция 1. Прочессы взаимодействия излучений и плазмы с твердым телом

\title{
МОДЕЛЬ ДИНАМИКИ ПРОХОЖДЕНИЯ ИОНОВ ЧЕРЕЗ ДИЭЛЕКТРИЧЕСКИЕ КАПИЛЛЯРЫ
}

\author{
Г.П. Похил, В.В. Чердынцев \\ НИияФ МГУ, Москва, Россия, pokhil1@yandex.ru
}

\begin{abstract}
При исследовании прохождения ионов через диэлектрические капилляры недавно было обнаружено, что при отличном от нуля угле входа ионов в капилляр проходящий ток возрастает не монотонно и совершает несколько осцилляций около оси капилляра прежде чем устанавливается стационарный режим и направление выходящего пучка ионов устанавливается вдоль оси капилляра. В настоящей работе предлагается модельное объяснение угловых осцилляций прошедшего пучка. В установившемся режиме в капилляре образуется одно заряженное пятно на стенке, которое отклоняет ионы и направляет их вдоль оси. Имеет место самоорганизация системы пучок - заряд стенки. Заряженное пятно вблизи входа капилляра автоматически образуется так, чтобы не происходило дальнейшей зарядки стенки. В начале прохождения пучка, пока это заряженное пятно еще не сформировалось, происходит зарядка более далеких от входа областей стенки капилляра. Благодаря резкой нелинейной зависимости тока утечки от плотности заряда на стенке, стекание этого удаленного заряда происходит с запаздыванием относительно времени формирования основного отклоняющего пятна, что и приводит к угловым осцилляциям проходящего пучка ионов.
\end{abstract}

\section{Введение}

Управление пучками ионов с помощью диэлектрических капилляров (guiding) имеет богатые перспективы применения, т.к. позволяет транспортировать быстрые ионы без потери энергии и без изменения заряда, и при этом не требует затрат энергии. Это явление интенсивно исследуется. Недавно обнаружено его новое, неизвестное ранее свойство. Оказалось, что при отличном от нуля угле входа пучка ионов в капилляр зарядка стенок капилляра происходит таким образом, что его прозрачность увеличивается немонотонно и, прежде чем устанавливается направление прошедшего пучка вдоль оси капилляра, пучок на выходе совершает несколько осцилляций по углу около направления оси [1]. Этот эффрект кажется необъяснимым на первый взгляд, т.к. естественно ожидать, что накопление заряда, который отклоняет пучок, на стенке капилляра происходит монотонно во времени. В настоящей работе дается простая модель, объясняющая осцилляции пучка.

\section{Основная часть}

Уравнение баланса заряда на стенке капилляра сложное для решения из-за того, что ток утечки сильно зависит от величины заряда:

$\frac{d q(x, t)}{d t}=j(x, t)-\sigma(q(x, t)) \cdot q(x, t)$

$q(x, t)$ - плотность заряда в точке $x, j(x, t)$ плотность тока, и $\sigma$ - проводимость для тока утечки. Здесь уравнение написано для наиболее простого случая, когда ток утечки в каждой точке определяется только плотностью заряда в этой точке. Уравнение имеет такой вид, если в монокапилляре утечка происходит через его стенку. В поликапилляре утечка происходит через торцы капилляра, и следует записывать интегродифференциальное уравнение. Мы будем рассматривать плоский монокапилляр для простоты анализа (это не уменьшит общность выводов работы). Схема управления пучком в установившемся режиме показана на рис. 1 [2]. Если уже установил- ся стационарный режим прохождения пучка через капилляр, то некоторая часть стенки $\ell$ заряжается до предельно возможной плотности заряда (при большей плотности ток утечки резко возрастает и заряд сбрасывается [2,3]), пучок отклоняется один раз и проходит через капилляр.

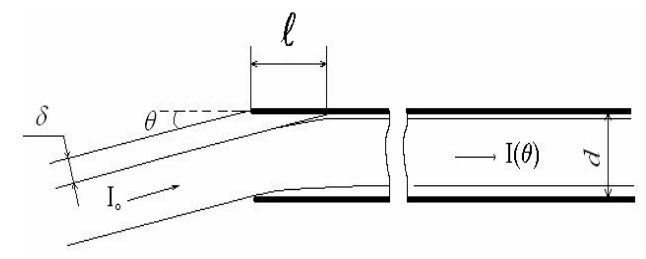

Рис. 1. Схема прохождения пучка. I - заряженная часть стенки в стационарном режиме.

Угол отклонения ионов в каждой точке $x$ можно записать так:

$$
\delta \theta(x, t)=\frac{\Delta p_{\perp}}{p}=\frac{\pi q(x, t) \cdot Z e}{E} \cdot \Delta x
$$

И полный угол отклонения на участке $\ell$ :

$$
\theta_{l}(t)=\frac{\pi Z e}{E} \int_{0}^{l} q(x . t) d x=\frac{\pi \cdot Q_{l}(t) \cdot Z e}{E}
$$

где $E$ - энергия иона, Z - заряд иона, и

$$
Q_{l}(t)=\int_{0}^{l} q(x, t) \cdot d x \text { - заряд участка } \ell .
$$

Но вначале прохождения пучка через капилляр заряд образуется не только на интервале $\ell$, но и за этим интервалом $\mathbf{Q}_{a d d}$. И только через некоторое время $t_{0}$, когда заряд на интервале $l$ становится достаточным для отклонения ионов на угол $\theta$, зарядка за интервалом $\ell$ прекращается (см. рис. 2). Ток утечки при плотности заряда меньше критической мал, поэтому этот заряд 
Секция 1. Процессы взаимодействия излучений и плазмы с твердым телом

живет дольше, чем заряд $Q_{\imath}$ достигает насыщения. Заряд за участком $\ell$ это интеграл $Q_{\text {add }}=\int_{l}^{L} q(x, t) d x$, и этот заряд отклоняет ионы на угол: $\theta_{a d d}(t)=\frac{\pi \cdot Q_{a d d}(t) \cdot Z e}{E}$. Угол отклонения пучка определяется полным зарядом капилляра:

$\theta(t)=\theta_{l}(t)+\theta_{\text {add }}(t)=\frac{\pi \cdot Z e}{E}\left(Q_{l}(t)+Q_{\text {add }}(t)\right)$. Зависимость от времени угла отклонения $\theta(t)$ такая же, как временная зависимость полного заряда $Q_{1}(t)+Q_{a d d}(t)$.

Уравнение (1) можно решить лишь численным способом, и это мы сделаем в дальнейшем. В данной работе сделаем модельное упрощение. Будем считать, что проводимость утечки не зависит от времени, но она различна на участке $\ell$ и за этим участком. Причем, естественно за участком она меньше, т.к. в реальности там меньше плотность заряда $\left(\sigma_{\text {add }}<\sigma_{\text {I) }}\right.$. Теперь можем записать простые модельные уравнения:

$$
\begin{aligned}
& \frac{d Q_{l}}{d t}=I_{l}-\sigma_{l} \cdot Q_{l} \\
& \frac{d Q_{a d d}}{d t}=\left\{\begin{array}{lll}
I_{a d d}-\sigma_{a d d} \cdot Q_{a d d} & \text { if } & t<t_{0} \\
-\sigma_{a d d} \cdot Q_{a d d} & \text { if } & t>t_{0}
\end{array}\right\}
\end{aligned}
$$

Из уравнения и рис. 2 ясен смысл параметра $t_{0}$. Это характерное время, за которое участок стенки капилляра $l$ заряжается практически до насыщения, т.е. прекращается поступление заряда на стенку за участком $\ell$.

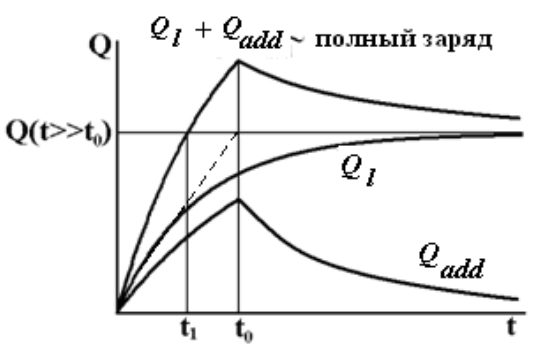

Рис. 2. Иллюстрация зависимости зарядов $Q_{1}$ и $Q_{a d d}$ от времени.

На третьем рисунке показано, как меняется траектория ионов в соответствии с изменением заряда на верхней пластине капилляра. В момент времени $t_{1}$ полный заряд становится равным заряду $Q_{\text {I }}$ при насыщении $\left(Q_{s a t}\right)$ и пучок выходит

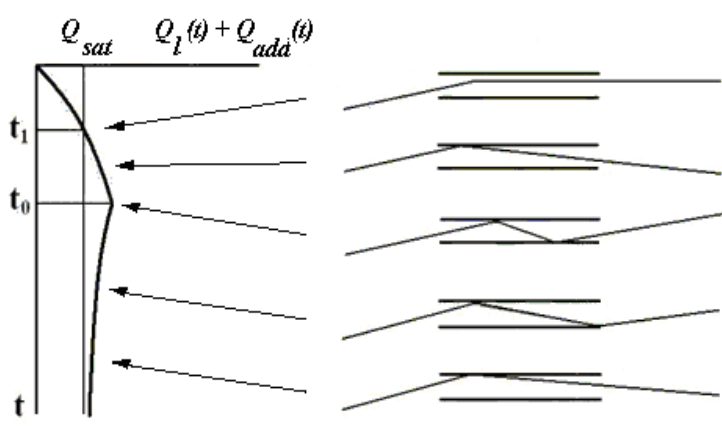

Рис. 3. Изменение траектории ионов с изменением величины заряда на пластине

из капилляра вдоль его оси. После момента $t_{1}$ полный заряд становится больше, чем $Q_{\text {I при }}$ насыщении и пучок отклоняется вниз от оси капилляра. И если в интервале времени между $t_{1}$ и $t_{0}$ полный заряд достаточно велик, то пучок коснется нижней пластины и начнет заряжать ее.

Этот заряд будет отклонять ионы вверх. После момента времени $t_{0}$ угол отклонения вниз от верхней пластины станет уменьшаться. Точка контакта пучка с нижней пластиной движется к выходу из капилляра. В какой-то момент пучок перестает касаться нижней пластины, но движется при этом с отрицательным углом. После этого направление пучка стремится к направлению оси капилляра вместе с стремлением полного заряда верхней пластины к $Q_{\text {sat }}($ см. Рис.2).

Возможно различное количество осцилляций пучка на выходе капилляра. Это определяется тем, насколько превышает полный заряд величину $Q_{\text {sat }}$ B момент $t_{\text {. }}$.

\section{Заключение}

Итак, можно сделать вывод, что осцилляции пучка возникают, благодаря запаздыванию разрядки $Q_{a d d}$ по отношению к достижению зарядом

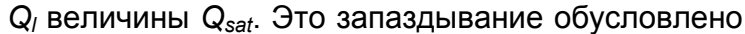
резкой зависимостью проводимости от плотности высаженного на поверхность заряда. Мы провели рассуждения для плоского капилляра. В цилиндрическом капилляре причина осцилляций пучка такая же. Работа выполнена при поддержке гранта РФФИ-11-02-001440а.

\section{Список литературы}

1. H.Q. Zhang, P. Skog, et. al. // Phys. Rev. - 2010. - A 82. - P. 052901.

2. Г.П. Похил, К.А. Вохмянина, Л.А. Жиляков, Т. lkeda, Y. Kanai, Y. Iwai, T.M. Kojima, Y. Yamazaki // Известия РАН, серия физическая. - 2008. - Т. 72. - 5. - С. 674-679.

3. N. Stolterfoht, et al // Phys. Rev. - 2007. - A 76. - P. 022712 .

\title{
MODEL OF THE GUIDING DYNAMICS
}

\author{
G.P. Pokhil, V.V. Cherdyntsev \\ SINP MSU, Moscow, Russia, pokhil1@yandex.ru
}

Recently it was created that the beam transmitted through insulator capillary makes a few angular oscillations around the capillary axes direction. It is surprising because due to self organization the charged pattern at the capillary wall is created by 
Секция 1. Прочессы взаимодействия излучений и плазмы с твердым телом

such manner, that the ions move along capillary axes after deviation from this pattern. Simple model of the beam angular oscillations at the insulator capillary exit is suggested in this work. Only one charged pattern exists when the stationary regime is achieved. But at the beginning of charging up the region behind this pattern also is charged up. The leakage current depends on charge density at the capillary wall very sharply. And this is the reason why small charge behind the pattern exists longer then the charge of the main pattern comes to saturation. So we have the retardation of discharging of the remote part in respect to basic pattern saturation, and the full charge exceed that is necessary for deviation the beam at the value of incident angle. The beam comes down from top side of capillary. When the extra charge of remote part disappears the beam comes back to the capillary axes. The beam makes more oscillations if the deviated beam touches down side of the capillary.

\title{
МАТЕМАТИЧЕСКИЕ МОДЕЛИ ФОРМИРОВАНИЯ ПЛАЗМЕННЫХ ПОТОКОВ ПРИ ЭЛЕКТРИЧЕСКОМ ВЗРЫВЕ ПРОВОДНИКОВ
}

\author{
В.Д. Сарычев \\ Сибирский государственный индустриальный университет, \\ ул. Кирова, 42, 654007, Новокузнецк, Россия, тел.8-951-597-3531, \\ e-mail: sarychev vd@physics.sibsiu.ru
}

Гетерогенные плазменные потоки, образованные при электрическом взрыве фольги с коаксиально расположенными электродами, способны ускорять порошки до скоростей порядка 1 км/с. При воздействии таких потоков на мишень происходит модификация поверхности, приводящая к повышению служебных характеристик изделия в несколько раз. Механизмы формирования гетерогенных потоков при коаксиальной геометрии электродов практически ранее не рассматривались. В настоящем докладе приведены методика съёмки разлета и динамика развития продуктов электрического взрыва фрольги при коаксиальном расположении электродов. Получены экспериментальные данные по формированию конфигурации разряда. Выявлены особенности развития границы разлета от напряжения зарядки конденсаторной батареи. Предложена схема формирования гетерогенного плазменного потока, заключающаяся в пространственном разделении разряда на центральную струю под электродом и кольцевую шайбу, замыкающую центральную струю и внешний электрод. Для динамики развития кольцевой шайбы предложена математическая модель движения плазмы, основанная на осреднении одномерных уравнений газовой динамики с учетом термодинамического, магнитного давления и сил сопротивления. Сравнения экспериментальных и расчетных зависимостей значений удовлетворительны в широком диапазоне параметров. Показано, что при напряжениях меньше 1 кВ происходит ускорение продуктов электровзрыва в термодинамическом режиме за счет высоких температур, а при напряжениях больше 1 кВ включается магнитодинамический режим ускорения.

\section{Введение}

Одним из перспективных импульсных методов является метод воздействия на материалы продуктами электрического взрыва проводников при коаксиально расположенных электродах. Продукты электровзрыва проводника содержат твердые частицы и газо-плазменную составляющую - гетерогенные плазменные потоки (ГПП). В область взрыва помещается порошок, а над электродами устанавливается направляющий канал. Благодаря высоким давлениям продуктов электрического взрыва частицы порошка имеют скорости порядка $1 \mathrm{kм} / \mathrm{c}$. Образовавшийся гетерогенный поток воздействует на поверхность обрабатываемой детали. Данный метод обработки позволяет вносить в поверхность различные порошки, и тем самым создавать соответствующую модифрикацию образца, благодаря которой служебные характеристики изделий могут повышаться в разы. Поэтому разработка фризических механизмов и математических моделей процессов, проходящих при формировании ГПП и их взаимодействия с твердыми телами, является актуальным.

\section{Экспериментальное изучение ГПП}

Для изучения свойств ГПП использовалась установка, включающая батарею импульсных конденсаторов, зарядное и разрядное устройства и плазменный ускоритель. Плазменный ускоритель выполнен в виде двух коаксиально расположенных цилиндрических электродов с замыкающей перемычкой, которая плотно прижата кольцомканалом перпендикулярно их осям. Особенностью выбора устройства для регистрации разлета плазмы торцевого плазменного ускорителя является характерное время протекания процесса порядка 100 мкс. На рис. 1 приведена принципиальная оптическая схема установки, включающая сверхскоростной фоторегистратор (СФР) с разверткой процесса по кадрам, разрядное устройство, позволяющее синхронизировать начало разлёта и съёмки.

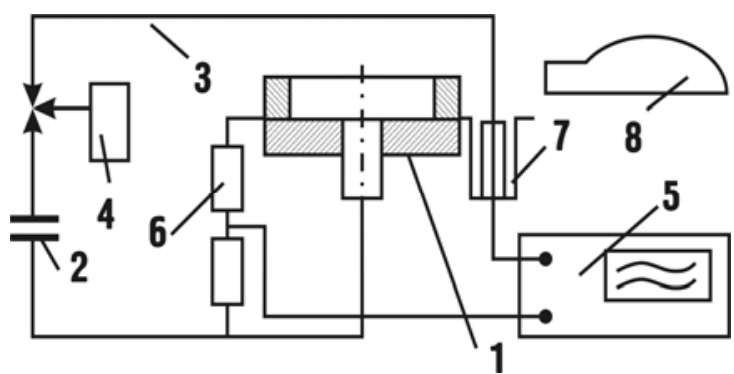

Рис. 1. Схема установки. 1 - плазменный ускоритель; 2 - батарея конденсаторов; 3 - форвакуумный разрядник; 4 - поджигающее устройство; 5 - запоминающий осциллограф; 6 - делитель напряжения; 7 - коаксиальный шунт;8 - СФР.

Съемка разлета плазменного потока осуществлялась перпендикулярно оси ускорителя через окошко в прижимном кольце. Эксперименты проводились в воздухе при атмосферном давлении. 
Фольги выбирались алюминиевые толщиной 10 мкм. В известных экспериментах СФР съёмка производилась при вылете плазмы из сопла или через прижимную шайбу. Это не позволяло зарегистрировать начало формирования потока.

В результате анализа съёмки можно выделить две характерные зоны в формировании потока (рис. 2). В первой зоне течение плазмы перпендикулярно оси $z$ c током $I_{1}$. Вторая зона расположена под центральным электродом, в которой происходит осевой разлет с током $I_{2}$. В первой зоне линии электрического тока параллельны диэлектрической шайбе. Зависимость логарифмма размеров первой зоны по $z$ от логарифма времени носит кусочно-линейный характер. Положение точки излома зависит от напряжения зарядки конденсаторной батареи. При напряжении на батарее < 1 кВ точка излома отсутствует, и указанная зависимость является прямолинейной. При напряжениях больше 1 кВ происходит отклонение от этой прямой в некоторый момент времени. Это отклонение связывается со сменой механизма ускорения, включаются силы магнитного давления. Следовательно, при напряжениях меньше $1 \mathrm{kB}$ работает тепловой режим разлета, при больших значениях напряжениях действует тепловое и магнитное давления. Эта смена механизмов отражена в математической модели.

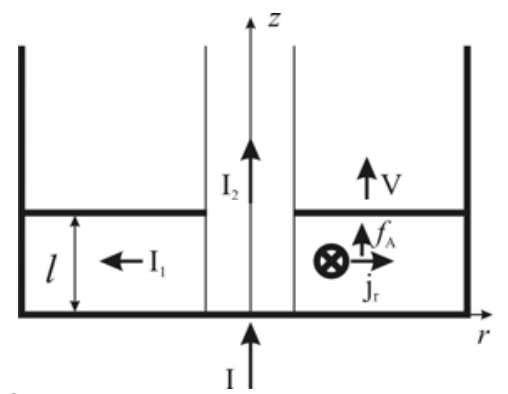

Рис. 2. Схема процесса разлета плазменного потока.

Для измерения зависимости силы тока от времени необходимо избавиться от электрических помех и добиться симметричного распределения токов. С этой целью был изготовлен медный цилиндрический бандаж, соединяющий крышку затворного устройства с корпусом. Сопротивление участка бандажа, на котором измерялось напряжение, составляло $4,4 \cdot 10^{7}$ Ом.

Измерения зависимости напряжения от времени проводились при помощи запоминающего осциллографра. Абсолютное значение тока рассчитывалось делением напряжения на сопротивление участка бандажа. При коротком замыкании в первом полупериоде зависимость силы тока от времени является синусоидой, а при переходе во второй полупериод происходит резкое уменьшение максимальной амплитуды, что связывается со скачкообразным изменением сопротивления форвакуумного разрядника (рис. 3). При коротком замыкании разрядного контура основное сопротивление сосредоточено в форвакуумном разряднике и в первом полупериоде пренебрежимо мало $\left(\mathrm{R}=10^{-7} \mathrm{Om}\right)$. Это позволило, используя фрормулы идеального колебательного контура провести расчеты параметров установки: ёмкость
$C=2,7$ мФ, индуктивность $L=0,084$ мкГн и полпериода разряда $T_{1 / 2}=47,5$ мкс. Имея емкость и индуктивность одного конденсатора ИМ-5-150: $\mathrm{C}_{0}=150$ мкФ, $\mathrm{L}_{0}=600$ нГН, можно определить индуктивность батареи 30нГн и подводящих шин 54,7 нГн. Полученные данные используются для расчетов параметров математической модели. Максимальные значения тока составляют 170 кА и 85 кА. Такое различие объясняется тем, что часть тока в виде плазменного фокуса (зона 2) выносится из области разряда. Из анализа полученных снимков видно, что в начальный момент времени (до 10мкс) в области центрального электрода образуется сфрерически расширяющаяся зона и в то же время происходит разряд между электродами. При времени больше 10мкс происходит формирование квазистационарной конфигурации со струёй в центре.

\section{Математическая модель разлета}

Характер течения и динамику первой зоны можно изучать теоретически, использую так называемую нульмерную математическую модель.

Процессы в плазменных ускорителях характеризуются сложным комплексом гидродинамических, тепловых и электромагнитных явлений, поэтому для математического моделирования необходимо привлекать систему уравнений нестационарной магнитной газовой динамики излучающей плазмы. Решение этих уравнений в силу своей сложности возможно только численными методами, которые бы позволили детально описать для торцевого плазменного ускорителя механизмы взаимодействия плазменного фрокуса (вторая зона ) с растекающимся перпендикулярно оси потоком (первая зона). Наличие свободной поверхности резко усложняет задачу даже для численного моделирования газодинамической задачи, и поэтому постановка и численное решение магнитоплазменной задачи является предметом специального исследования.

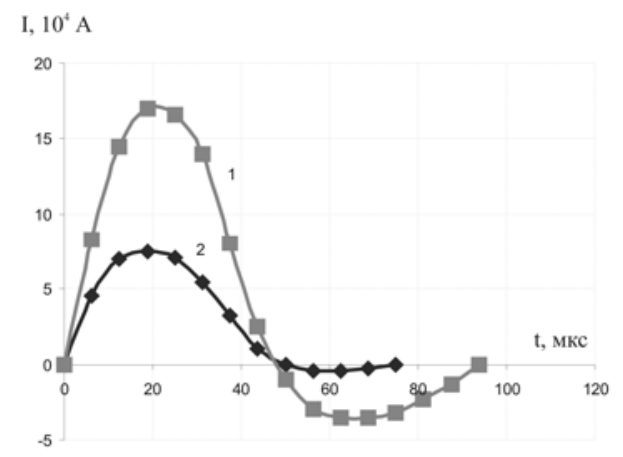

Рис. 3. Зависимость силы тока от времени. 1 - короткое замыкание; 2 - пробой фольги при напряжении 1 кВ.

При моделировании плазменных потоков сложились определенные приемы и модели, связанные с упрощением исходной системы уравнений и позволяющие связать параметры установки с параметрами плазменных потоков. Такие модели для коаксиального ускорителя («модель шайбы») и для ускорителя с напуском газа («модель сне- 
Секиия 1. Проиессы взаимодействия излучений и плазмы с твердым телом

гоочистителя») были предложены в 60-х годах, с помощью которых проведен ряд оптимизационных исследований. Для торцевого ускорителя, который рассматривается в данной работе, упрощенная модель ранее не предлагалась.

Ниже рассмотрим упрощенную задачу движения потока и разряда для торцевого ускорителя. На эту роль подходит исследование динамики зоны вне электродов (первой зоны на рис. 2). В качестве модели выбираем движение плазменного кольца-шайбы, внутренний диаметр которого коммутирует со струёй, а внешний - с периферийным электродом установки. Задача состоит в расчете параметров плазменной перемычки, движущейся между коаксиальными электродами под действием собственного магнитного поля и газодинамического давления. Ниже получим уравнения движения и уравнение Кирхгофра, а начальные условия выберем с учетом специфики нашей ситуации.

Силу тока батареи можно представить $l=l_{1}+l_{2}$. На этом предположении основано объяснение различия в амплитудных значениях тока через взрывающуюся фольгу и деформируемую пластину при коротком замыкании. Поэтому это предположение может быть использовано в построении приближенной математической модели. Для простоты принимаем, что давление зависит от средних значений температуры и плотности, а сила сопротивлению движению пропорциональна квадрату скорости.

В качестве исходных уравнений модели выбираем законы изменения импульса и массы и закон Кирхгофа:

$$
\begin{aligned}
& \frac{\partial \rho}{\partial t}=-\frac{\partial \rho V}{\partial z}, \frac{\partial \rho V}{\partial t}=-\frac{\partial \rho V^{2}}{\partial z}-\frac{\partial p}{\partial z}+f_{A}, \\
& Q / C_{0}+\frac{d}{d t}\left(L_{0} I+\Phi\right)+\left(R_{0} I+R_{p} I_{1}+U_{E}\right)=0 .
\end{aligned}
$$

где $V$-скорость вдоль оси z, $\rho$ - плотность плазмы, $p$-давление, $f_{A}$ - объемная плотность силы Ампеpa, Q-заряд на батареи во время разряда, и параметры задачи: $C_{0}, L_{0}, R_{0}$ - емкость батареи, индуктивности и сопротивления батареи, $\Phi, R_{\mathrm{p}}$ магнитный поток плазменной шайбы и еe сопротивление, $U_{0}, U_{\mathrm{E}}$-напряжение на батареи, индуцированное в плазме напряжение. Определим интегральные характеристики течения импульс, массу шайбы, силу Ампера, магнитный поток плазменной шайбы и ее сопротивление, индуцированное в плазме напряжение за счет ее движения по фрормулам:

$$
\begin{aligned}
P & =\int_{0}^{l} \int_{a}^{b} \rho V(z, r, t) 2 \pi r d r d z ; \quad M=\int_{0}^{l} \int_{a}^{b} \rho(z, r, t) 2 \pi r d r d z \\
F_{A} & =\int_{0}^{l} \int_{a}^{b} j_{r} B_{\varphi}(z, r, t) 2 \pi r d r d z ; U_{E}=\int_{0}^{l} \int_{a}^{b} V B_{\varphi}(z, r, t) 2 \pi r d r d z \\
\Phi & =\int_{0}^{l} \int_{a}^{b} B_{\varphi}(z, r, t) 2 \pi r d r d z ; R=\left(\int_{0}^{l} \int_{a}^{b}\left(j_{r}(z, r, t) 2 \pi / \sigma\right) 2 \pi r d r d z\right) / I
\end{aligned}
$$

Усредняя первые два уравнения (1) по пространству, получим уравнения движения и изменения массы для всей шайбы как целого:

$$
\frac{d P}{d t}=F_{A}-\rho_{0} V^{2} S+R T \frac{M}{l \mu}, \quad \frac{d M}{d t}=0 .
$$

При выводе уравнений (2) воспользовались: $V(0, t)=0$, уравнением идеального газа $p(0, t)=R T \rho / \mu$ и законом сопротивления в виде $p(l, t)=\rho_{0} V^{2}$. Здесь $\rho_{0}, T, \mu, R$ - соответственно плотность окружающей среды, температура и молярная масса плазмы, универсальная газовая постоянная. Выражения для давления отражают характер сопротивления движению плазменного сгустка и газодинамическое давление за счет высокой температуры. Из (2) следует, что масса шайбы остается постоянной. Принимая, что распределение скорости по оси будет треугольным с максимумом $V$ при $z=I$, и $F_{A}=A l_{1} I_{2}$, где $A=\mu_{0} \ln (a / b) / 2 \pi, a, b$ - геометрические размеры соответственно периферийного и цен нулем при $z=0$,то можно получить $P=M V / 2$. Для получения выражения для силы Ампера $F_{\mathrm{A}}$ считаем, что токи по зонам пропорциональны току батареи $l_{1}=k l, l_{2}=(1-k) l$ и однородны, тогда получаем трального электродов. Экспериментальная зависимость силы тока в первом полупериоде близка к синусоидальной, поэтому проведен анализ системы (2) без учета уравнения Кирхгофа. Введем обозначения

$$
f_{1}=\frac{2 A k(k-1) t_{0} I_{0}^{2}}{M V_{0}}, f_{2}=\frac{2 R T}{\mu V_{0}^{2}},
$$

где $t_{0}, V_{0}, I_{0}$ - характерные значения времени, скорости и силы тока. Систему уравнений (2) можно свести к безразмерному виду

$$
\frac{d V}{d t}=f_{1} \cdot \sin ^{2}(t)+\frac{f_{2}}{z}-2 \cdot V^{2}, \quad \frac{d z}{d t}=V .
$$

Таким образом, получена двухпараметрическая математическая модель $\left(f_{1}, f_{2}\right)$. Численное решение (3) с нулевыми начальными условиями позволило выявить участки линейной зависимости $\ln (z)$ от $\ln (t)$, близкие к экспериментальной (рис.4).

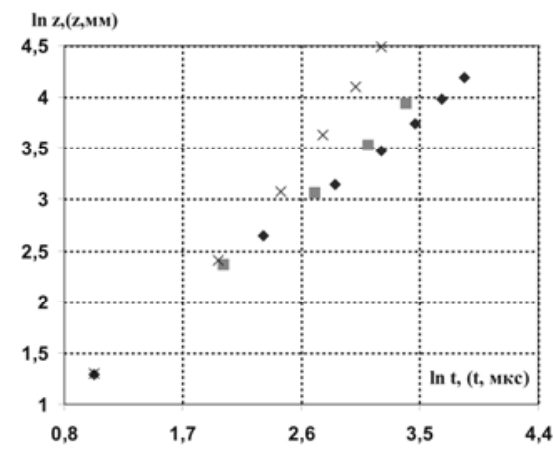

Рис. 4. Моделирование движения границы первой зоны. При - $f_{1}=6, f_{2}=1 ;$ - $f_{1}=20, f_{2}=1 ; x-f_{1}=80, f_{2}=1$.

\section{Заключение}

В результате предложенной экспериментальной методики по регистрации разлета плазмы электрического взрыва фольги при коаксиальном подводе электродов удалось наблюдать процесс разлета. Анализ экспериментальных данных позволил предложить схему разлета, на основании 
Секция 1. Прочессы взаимодействия излучений и плазмы с твердым телом

которой получена двухпараметрическая математическая модель, адекватно отражающая экспериментальные данные. Сказанное позволяет заявить о выявленном механизме формирования гетерогенных плазменных потоков при электрическом взрыве фольги в указанной геометрии.
Работа выполнена при финансовой поддержке ФЦП «Научные и научно-педагогические кадры инновационной России» на 2009-2013 ге. (гос. контракт № 14.740.11.0813) и грантом РФФИ (проект № 11-0291150-ГФЕH-a).

\title{
MATHEMATICAL MODELS OF FORMATION OF PLASMA STREAMS AT ELECTRIC EXPLOSION OF CONDUCTORS
}

\author{
Vladimir D. Sarychev \\ Siberian State Industrial University, Ru-654007, Russian, Novokuznetsk, Kirov street, 42, \\ tel.: 8-951-597-3531, e-mail: sarychev vd@physics.sibsiu.ru
}

\begin{abstract}
Plasma accelerators find wide application in surface updating. Mechanisms of formation of plasma streams and their influence on a surface of materials are well studied. The heterogeneous plasma streams formed at electric explosion of a foil with at coaxial geometry of electrodes are capable to accelerate powders till speeds of an order of $1 \mathrm{~km} / \mathrm{s}$. At influence of such streams on a surface of materials there is the updating of a surface leading to increase of office characteristics of a product in times. Mechanisms of formation of heterogeneous streams at coaxial geometry of electrodes practically were not considered earlier. In the present report the technique of shooting of scattering and dynamics of development of products of electric explosion of a foil and strips are resulted at a coaxial arrangement of electrodes. Experimental data on formation of a configuration of the category are received. Features of development of border of scattering from pressure of gymnastics of the condenser battery are revealed. The scheme of formation of the heterogeneous plasma stream, consisting in spatial division of the category into the central stream under an electrode and the ring washer closing the central stream and an external electrode is offered. For dynamics of development of a ring washer the mathematical model of movement of the plasma, based on averaging of the onedimensional equations of gas dynamics taking into account thermodynamic, magnetic pressure and forces of resistance is offered. Comparisons of experimental and settlement dependences of values are satisfactory in a wide range of parameters. It is shown that at voltage less $1 \mathrm{kB}$ there is an acceleration of products of electro explosion in a thermodynamic mode at the expense of heats, and at voltage more $1 \mathrm{kB}$ joins electromagnetic pressure an acceleration mode. Experimental data and mathematical model about formation of superficially periodic structures at influence of heterogeneous plasma streams are results.
\end{abstract}

